\title{
Efecto de la remineralización de lesiones cariosas incipientes de un barniz de flúor con fosfato tricálcico
}

\section{Remineralization effect on incipient carious lesions of a sodium fluoride with tricalcium phosphate varnish}

\author{
María Lilia Adriana Juárez-López, ${ }^{1}$ María del Pilar Adriano-Anaya, ${ }^{2}$ Nelly Molina-Frechero, ${ }^{3}$ Francisco Murrieta- \\ Pruneda ${ }^{4}$
}

\section{Resumen}

OBJETIVO: Evaluar la efectividad preventiva de la aplicación de un barniz de fluoruro de sodio enriquecido con fosfato tricálcico en escolares de alto riesgo cariogénico.

MÉTODO: Ensayo clínico efectuado durante 12 meses en escolares de seis años inscritos en una escuela primaria en la Ciudad de México. Los sujetos de estudio se asignaron al azar a dos grupos: a) barniz de fluoruro de sodio con trifosfato cálcico cada 4 meses, más cepillado dental con dentífrico fluorurado; $b$ ) sólo cepillado dental con dentífrico fluorurado. Todos los participantes recibieron asesoría acerca de la técnica de cepillado. Se registraron los índices de caries por superficie en dientes primarios y permanentes y se calculó la fracción preventiva. Mediante el Sistema Internacional de Detección y Evaluación de Caries se identificaron los molares con lesiones cariosas incipientes. Para la comparación de los grupos se aplicaron las pruebas t de Student, t pareada, U de Mann Whitney y Wilcoxon.

RESULTADOS: De 106 escolares que terminaron los tratamientos, el grupo que recibió barniz de fluoruro de sodio con fosfato tricálcico tuvo menor incremento de superficies cariadas, perdidas u obturadas en dientes permanentes que el grupo de control $(p=$ 0.087). La fracción de protección del barniz fue de $36 \%$ para los molares permanentes. En el seguimiento de las lesiones cariosas incipientes se observó que $15 \%$ del grupo que recibió barniz de fluoruro de sodio con fosfato tricálcico se recuperó en comparación con $0.4 \%$ del grupo control $(p=0.001)$.

CONCLUSIONES: La aplicación cuatrimestral de barniz de fluoruro de sodio con fosfato tricálcico demostró ventajas preventivas, reversión y evitó el avance de las lesiones cariosas incipientes.

PALABRAS CLAVE: Lesiones cariosas; barniz de fluoruro de sodio; fosfato tricálcico; niños; dentífricos; fosfatos cálcicos.

Abstract

OBJECTIVE: To determine the preventive and remineralization effectiveness of carious lesions with the topical application of a Sodium Fluoride varnish with Tricalcium Phosphate.

METHOD: A clinical trial was conducted with 106 schoolchildren of 6 years enrolled in a primary school in Mexico City for 12 months. Children were randomly assigned in two groups: a) Sodium Fluoride with Calcium Trifosphate varnish every 4 months and dental brushing fluoridated dentifrice; b) Only dental brushing with fluoridated dentifrice. All the participants received brushing technique instructions. The caries indices by surface in primary and permanent teeth were recorded: $\mathrm{dmfs}$, DMFS and the preventive fraction was calculated. In addition, the molars with incipient carious lesions were detected using the International Caries Detection and Evaluation System. For the comparison of the groups, student T, paired T, U of Mann Whitney and Wilconson tests were applied.
${ }^{1}$ Profesor de posgrado en Estomatología del niño y del adolescente. 2 Profesor FES Zaragoza.

${ }^{3}$ Profesor, División de Ciencias de la Salud. UAM Xochimilco.

${ }^{4}$ Profesor de posgrado.

Facultad de Estudios Superiores Zaragoza, UNAM, Ciudad de México.

Recibido: 4 de octubre 2017

Aceptado: 7 de marzo 2018

Correspondencia

María Lilia Adriana Juárez López liadju@yahoo.com

Este artículo debe citarse como Juárez-López MLA, Adriano-Anaya MP, Molina-Frechero N, Murrieta-Pruneda F. Efecto de remineralización de lesiones cariosas incipientes de un barniz de flúor con fosfato tricálcico. Acta Pediatr Mex. 2018;39(5):263-270. 
RESULTS: The varnish group had a lower DMFS increment than the control group $(\mathrm{p}=$ 0.087 ). The protection fraction for the varnish was $36 \%$ for the permanent molars. Following the incipient carious lesions, it was observed that $15 \%$ of the NaF-TPC varnish group recovered compared to $0.4 \%$ of the control. $(p=0.001)$.

CONCLUSION: The application of NaF-TCP varnish every four months in a year showed preventive benefits as well as to reverse and prevent the advance of incipient carious lesions.

KEY WORDS: Carious lesions; Sodium Fluoride varnish; Tricalcium Phosphate; Child; Dentifrices. Calcium Phosphates.

\section{ANTECEDENTES}

En México, la caries dental afecta a los niños desde edades tempranas, lo que incrementa su prevalencia y severidad conforme avanza la edad. Se ha informado que, durante el periodo de dentición mixta, $77 \%$ de los niños de 6 a 9 años tienen 3.8 dientes primarios y de 0.7 a 1.3 dientes permanentes cavitados, obturados o perdidos por estas causas. ${ }^{1}$

En la etapa inicial la lesión cariosa puede pasar clínicamente inadvertida con la apariencia de una mancha blanca. En la fase inicial debido a la pérdida mínima de minerales la capa superficial de la estructura dentaria intacta se colapsa y produce una cavidad. ${ }^{2}$ La importancia de la detección oportuna, y de la aplicación de tratamientos preventivos y de mínima invasión es evitar, detener o revertir el proceso carioso. Entre las estrategias para prevenir la lesión cariosa está la aplicación de flúor en diferentes presentaciones sistémicas y tópicas: geles, colutorios, pastas, espumas y barnices. ${ }^{3}$

Los barnices de flúor poseen una concentración elevada del elemento de 5000 ppm y propician un contacto prolongado con la superficie dentaria. En años recientes se han enriquecido con compuestos a base de calcio para abastecer con iones minerales la interfase de esmalte y así evitar o revertir la desmineralización.

Al respecto existen antecedentes favorables derivados de investigaciones in vitro, aunque los reportes clínicos de los barnices de flúor sin componentes de calcio son controversiales. Hay clínicos que señalan reducciones de $46 \%$ en la incidencia de caries después de aplicaciones durante dos o tres años, otros trabajos no encontraron ventajas adicionales..$^{4,5,6}$ La incorporación de compuestos cálcicos a los barnices de flúor requiere evidencias clínicas que contemplen los determinantes biológicos y sociales de nuestra población.

La salud bucal de los escolares requiere programas preventivos efectivos que disminuyan la afectación por caries dental. A nivel comunitario, en nuestro país se implementó, desde 1993, la fluoración de la sal de mesa. Además, a través del programa de salud bucal escolar se aplican colutorios quincenales de fluoruro de sodio al $0.02 \%{ }^{7}$ A pesar de esto, las necesidades de tratamientos de restauración y pulpares derivados del daño de la caries dental son crecientes, por lo que es relevante contar con evidencias de la aplicación de nuevas estrategias dirigidas, además de la prevención, a la intervención temprana del proceso carioso. 
El objetivo de este trabajo fue: evaluar la efectividad preventiva de la aplicación de un barniz de fluoruro de sodio enriquecido con fosfato tricálcico en escolares de alto riesgo cariogénico.

\section{MATERIALES Y MÉTODOS}

Ensayo clínico efectuado durante 12 meses en escolares de seis años inscritos en una escuela primaria en la Ciudad de México. El protocolo cumplió con los lineamientos de bioética del comité de investigación de nuestra institución.

Se obtuvo el consentimiento firmado de los padres de los niños después de explicarles los objetivos de la investigación. Criterios de inclusión: escolares de uno y otro sexo, con dentición mixta, que contaran con el consentimiento informado firmado por su padre o tutor y que tuvieran, al menos, una lesión cariosa incipiente en los molares. Criterios de exclusión: casos con defectos estructurales del esmalte o con algún aparato de ortodoncia. Criterio de eliminación: pacientes que no cumplieron con las sesiones del programa.

Los escolares participantes se distribuyeron al azar, por conglomerados en dos grupos: el grupo A recibió tres aplicaciones del barniz de fluoruro de sodio enriquecido con fosfato tricálcico con un intervalo de cuatro meses y asesorías mensuales acerca de la técnica de cepillado. El grupo B o control solo recibió las asesorías mensuales referentes a la técnica de cepillado. A todos los escolares se les indicó cepillarse con un dentífrico con fluoruro de sodio.

El diagnóstico basal y final lo estableció un clínico, previamente estandarizado con un coeficiente de kappa de 0.85 para los índices de superficies cariadas, pérdidas y obturadas de dientes permanentes y primarios (CPOS, cpos respectivamente), y un valor de kappa de 0.82 en los criterios del Sistema Internacional de Detección y Valoración de Caries (ICDAS). ${ }^{8,9}$ El clínico a cargo de establecer el diagnóstico de lesiones cariosas ignoraba a qué grupo pertenecían los niños; es decir, desconocía el tratamiento al que pertenecía cada niño. Las revisiones se programaron al azar y en días diferentes a la implementación de los programas. Esto se logró con fichas separadas: una para los diagnósticos y otra para el control de asistencia a las sesiones preventivas. Luego de identificar los índices CPOS y cpos por paciente se identificaron y registraron en cada escolar los molares permanentes o primarios libres de caries o con lesión cariosa incipiente de acuerdo con los criterios 0,1 y 2 de ICDAS. Cuadro 1

Después del cepillado dental se efectuó una revisión clínica con ayuda de espejos planos del número 5 y sondas con punta redonda bajo luz blanca artificial. Para la determinación de lesiones cariosas incipientes se secaron las superficies dentarias con jeringa triple, durante cinco segundos. Todos los procedimientos clínicos se llevaron a cabo siguiendo las medidas de bioseguridad requeridas.

Los índices epidemiológicos de caries basales y la distribución de los molares sanos o con lesiones incipientes de caries se muestran en los Cuadros 2 y 3 sin diferencias estadísticamente significativas entre los grupos en la etapa basal. El flujograma del estudio se muestra en la Figura 1, donde se observa que de los 116 escolares que iniciaron el programa se eliminaron 10 por no cumplir con las sesiones preventivas o por baja. Solo se consideraron 106 para la evaluación final.

La aplicación de los tratamientos la efectuaron otros tres odontólogos diferentes al responsable del diagnóstico, quienes previamente recibieron una capacitación específica para la aplicación del barniz de NaF-TCP (Clinpro ${ }^{\text {TM }}$ barniz blanco con 22000 ppm de flúor y fosfato tricálcico), que 
Cuadro 1. Criterios del Sistema Internacional para Detección de Caries (ICDAS) ${ }^{9}$

\begin{tabular}{|c|c|}
\hline 0 & Superficie dental sana. No hay evidencia de caries después de la aspiración (desecación durante cinco segundos). \\
\hline 1 & $\begin{array}{l}\text { Primer cambio visual en el esmalte (zona con mayor opacidad, lesión blanca o marrón) detectado después de la } \\
\text { desecación de la superficie del área durante cinco segundos. }\end{array}$ \\
\hline 2 & $\begin{array}{l}\text { Cambio distintivo blanco o marrón en el esmalte, visto sin necesidad de la desecación y que se extiende más allá } \\
\text { del área de la fisura. }\end{array}$ \\
\hline 3 & Microcavidad resultado de la fractura del esmalte por pérdida mineral. No se aprecia dentina en la zona de la fisura. \\
\hline 4 & $\begin{array}{l}\text { Cambio interno de la superficie del esmalte. Se observa una sombra oscura semiescondida de la dentina, con o } \\
\text { sin esmalte fracturado. }\end{array}$ \\
\hline 5 & Cavidad distintiva que abarca el esmalte y la dentina que se observa en la base. \\
\hline 6 & Cavidad extensa con dentina visible en la base y paredes de la cavidad. \\
\hline
\end{tabular}

Cuadro 2. Índices de experiencia de caries por superficie antes de la aplicación de tratamientos preventivos

\begin{tabular}{l|c|c|c|} 
& Barniz & Control & Valor de $\mathbf{p}$ \\
\hline CPOS $^{*}$ & $0.61 \pm 1$ & $0.34 \pm 0.7$ & 0.3 \\
cpos $^{* *}$ & $8 \pm 9$ & $11.1 \pm 10$ & 0.07 \\
\hline
\end{tabular}

*Superficies cariadas, perdidas u obturadas en dientes permanentes.

**Superficies cariadas, perdidas u obturadas en dientes primarios.

Cuadro 3. Distribución de molares sanos y con lesión cariosa incipiente en los grupos de estudio (basal)

\begin{tabular}{l|c|c|}
\hline Grupo & SLC & LCI \\
\hline $\begin{array}{l}\text { Barniz } \\
\mathrm{n}=461\end{array}$ & $230(49.9 \%)$ & $231(50.1 \%)$ \\
$\begin{array}{l}\text { Control } \\
\mathrm{n}=360\end{array}$ & $165(45.8 \%)$ & $195(54.2 \%)$
\end{tabular}

U de Mann Whitney: grupo barniz versus grupo control, $p=0.2$

SLC: $\sin$ lesión cariosa (ICDAS $=0$ ).

LCI: lesión cariosa incipiente (ICDAS = 1y 2).

se realizó después del cepillado por cuadrantes, con la ayuda de un pincel y previo aislamiento relativo con rollos de algodón. Se utilizó la presentación de dosis individual de $0.50 \mathrm{~mL} .{ }^{10} \mathrm{Se}$ solicitó a los escolares no ingerir alimento por un lapso de 60 minutos después de la aplicación, y no cepillar los dientes en 12 horas. La

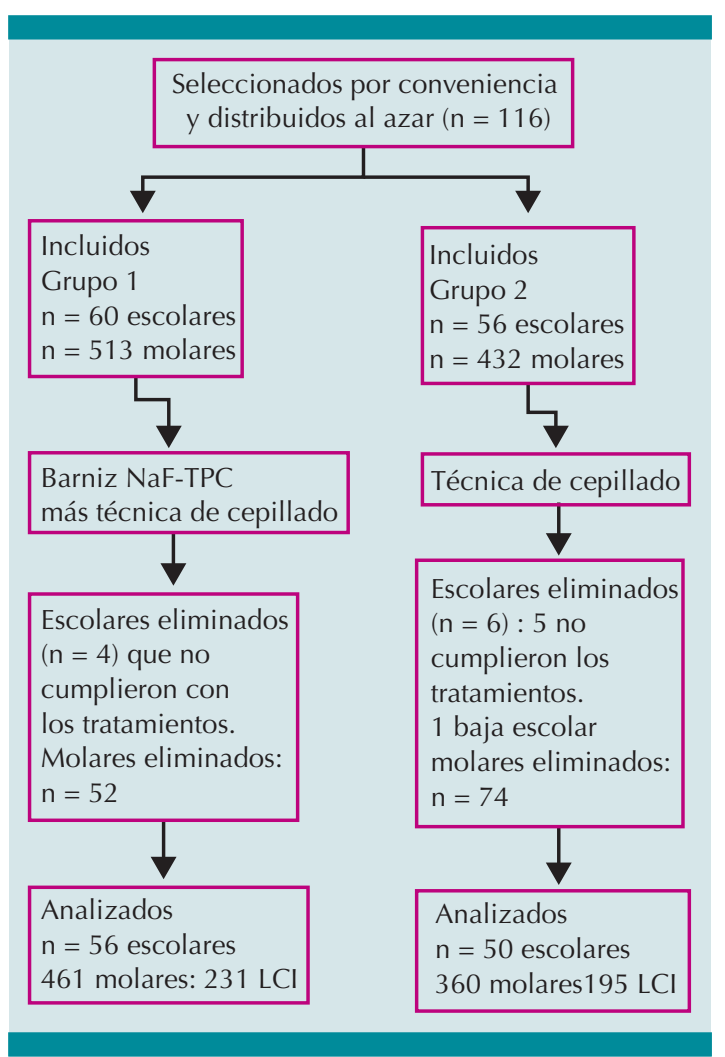

Figura 1. Flujograma del ensayo clínico: LCl: lesión cariosa incipiente.

técnica de cepillado de barrido derivada de la técnica de Stillman modificada ${ }^{11}$ se explicó en grupos de cinco con la ayuda de un tipodonto. Se efectuaron sesiones mensuales de promoción del 
autocuidado bucal, se obsequiaron cepillos dentales y pastas de 1450 ppm de flúor (Colgate total con fluoruro de sodio [1450 ppm de flúor]) para sus casas y con la ayuda de pastillas reveladoras se llevó a cabo una supervisión individualizada para mostrar al niño las zonas que requerían refuerzo del cepillado. El apego al cepillado se corroboró semanalmente preguntando a los participantes si habían seguido las indicaciones.

Para el análisis estadístico de los datos se utilizó el paquete estadístico SPSS V.15.0. Para la descripción de las variables cuantitativas se calculó el promedio y desviación estándar. Los valores cuantitativos se compararon con la prueba de $t$ de Student entre los diferentes grupos y la t pareada para comparar el antes y después de cada grupo. La fracción de prevención de caries de la aplicación del barniz se calculó mediante la diferencia entre los valores de los índices CPOS y cpos del grupo control con respecto a los valores del grupo de barniz entre la diferencia del grupo control y por $100:{ }^{12} \mathrm{PF}=100\left(\mathrm{D}_{\mathrm{C}}-\mathrm{D}_{\mathrm{T}} / \mathrm{D}_{\mathrm{C}}\right)$.

Se calcularon frecuencias y proporciones de los molares sanos y con lesiones incipientes y para su comparación del antes versus después en cada grupo de tratamiento se aplicó la prueba de Wilconson; así como entre grupos con la prueba U de Mann-Whitney.

\section{RESULTADOS}

De 106 escolares que terminaron los tratamientos, el grupo que recibió barniz de fluoruro de sodio con fosfato tricálcico tuvo menor incremento de CPOS que el grupo control $(p=0.087)$. En el Cuadro 4 se muestran los índices CPOS y cpos finales, así como el factor de protección. Se encontró que el incremento del CPOS fue menor en el grupo que recibió el barniz, sin diferencias significativas entre los grupos.

De los 821 molares detectados sanos (SLC) y con lesiones cariosas incipientes (LCI): 395 SLC y 426
Cuadro 4. Índices de experiencia de caries por superficie y factor de prevención (FP) después de la aplicación de tratamientos preventivos

\begin{tabular}{|l|c|c|c|c|} 
& Barniz & Control & Valor de $\mathbf{p}^{\mathbf{1}}$ & FP' $^{2} \%$ \\
\hline CPOS $^{3}$ & $1.4 \pm 2$ & $2.2 \pm 2$ & 0.08 & 36 \\
\hline Cpos $^{4}$ & $10 \pm 10$ & $12 \pm 10$ & 0.2 & 16 \\
\hline
\end{tabular}

1 Prueba t de Student.

${ }^{2}$ Fración de prevencion de caries.

${ }^{3}$ Superficies cariadas, perdidas u obturadas en dientes permanentes.

${ }^{4}$ Superficies cariadas, perdidas u obturadas en dientes primarios.

con $\mathrm{LCl}$, en el Cuadro 5 se incluyen los cambios posintervención en los molares diagnosticados solo con lesión cariosa incipiente. El tamaño del efecto para el grupo de barniz fue de 0.15 , pues 15\% (IC95\%: 19-25) se recuperó en comparación con solo $4 \%$ del grupo control (IC95\%: 1-6). El porcentaje de molares que evolucionaron a lesión cariosa cavitada (LCC) fue mayor en el grupo control $(p=0.0001)$.

Con respecto a los molares diagnosticados sin lesión cariosa (SLC) al inicio del estudio se encontró que en el grupo barniz $(n=230)$, después de la intervención, 135 (59\%) permanecieron sanos, 64 (28\%) tenían lesión cariosa incipiente

Cuadro 5. Cambios en los molares con lesiones de caries incipientes después de la intervención preventiva en escolares

\begin{tabular}{l|cccc}
\hline Basal & \multicolumn{4}{|c}{ Posterior al tratamiento } \\
\hline Sólo LCl & SLC & LCl & LCC & Valor $\mathrm{p}^{1}$ \\
\hline Barniz & 35 & 151 & 45 & 0.000 \\
$\mathrm{n}=231$ & $(15.2 \%)^{\mp}$ & $(65.3 \%)$ & $(19.5 \%)^{2}$ & \\
Control & $8(4.1 \%)^{\mp}$ & 122 & 65 & 0.000 \\
$\mathrm{n}=195$ & & $(62.6 \%)$ & $(33.3 \%)^{2}$ & \\
\hline
\end{tabular}

1 Prueba de Wilcoxon (basal versus después de la intervención en cada grupo).

${ }^{2}$ Prueba U de Man Whitney: grupo barniz versus grupo control $(p=0.001)$.

SLC: sin lesión cariosa.

LCl: lesión cariosa incipiente.

LCC: lesión cariosa cavitada. 
y 31(14\%) avanzó a lesión cavitada en comparación con el grupo control $(n=165)$ donde 105 (64\%) de los molares permanecieron sanos; 38 (23\%) tenían lesión incipiente y 22 (13.4\%) avanzaron a la cavidad. La comparación del antes y después entre el grupo barniz y control, considerando los molares sanos, no mostró diferencias estadísticamente significativas entre los grupos $(p=0.343)$.

\section{DISCUSIÓN}

La implementación de estrategias dirigidas a conservar la salud dental es uno de los principales retos de la práctica odontopediátrica puesto que la caries dental es la enfermedad bucal más frecuente en los niños, que afecta la calidad de vida no solo por los cuadros infecciosos y dolorosos, sino también por las alteraciones en la función, el sueño y en el comportamiento. ${ }^{13}$

En esta investigación, luego del seguimiento durante un año de un programa preventivo, se observó que la aplicación de barniz de fluoruro de sodio enriquecido con fosfato tricálcico aportó un factor de prevención de $36 \%$ para los molares permanentes y de $16 \%$ para los primarios, con un incremento menor de superficies cavitadas, perdidas u obturadas que el grupo control. Estos hallazgos son similares al $40 \%$ informado por Arruda ${ }^{12}$ y con Marino, quien, al analizar diferentes vehículos para la aplicación tópica de flúor destacó como ventaja de los barnices con alta concentración de flúor una protección promedio de $33 \% .{ }^{14}$

Los nuevos tratamientos basados en la combinación de flúor con compuestos de calcio y fosfato han marcado una nueva tendencia en la odontología de mínima invasión, bajo la premisa de detener y revertir el proceso carioso en su fase inicial.

En esta investigación, además de observar los efectos preventivos de la aplicación cuatrimes- tral del barniz de fluoruro de sodio enriquecido con fosfato tricálcico en escolares mexicanos, es relevante señalar que se encontró recuperación de $15 \%$ de los casos de lesiones cariosas incipientes, mucho mayor que en el grupo control. Lo anterior vislumbra las posibilidades de una intervención en fases iniciales del proceso carioso para evitar los tratamientos de restauración.

El proceso natural de la recuperación de la desmineralización coexistente en las lesiones cariosas incipientes está determinado, en gran medida, por la cantidad y composición salival que equilibra el $\mathrm{pH}$ y actúa como reservorio de minerales: si el $\mathrm{pH}$ en la interfase del esmalte aumenta, baja la concentración de hidrógenos y los iones hidroxilo, calcio y fosfatos quedan disponibles para reconstruir los prismas del esmalte dañados, produciéndose así la remineralización de manera fisiológica. ${ }^{15,16}$

El principio del barniz de fluoruro de sodio enriquecido con fosfato tricálcico es aportar y saturar a la superficie del esmalte elementos minerales que favorezcan la reparación de las zonas desmineralizadas. Gao S y su grupo, ${ }^{17}$ mediante una revisión sistemática, insistieron en el efecto remineralizante del fluoruro de sodio en barniz. Rirattanapong y colaboradores, ${ }^{18}$ en un estudio in vitro, encontraron inhibición del progreso de caries incipientes en dientes primarios. Elkassas y sus coautores mostraron que el trifosfato cálcico incrementa la microdureza del esmalte proporcionándole una resistencia al ataque ácido mayor a lo observado en barnices compuestos que sólo contienen fluoruro. ${ }^{19}$

El barniz de flúor favorece la alcalinidad del $\mathrm{pH}$ salival, ${ }^{3}$ disminuye por una semana la conformación de la biopelícula dentaria, sobre todo de colonias de Streptococcus mutans..$^{20}$ Después de la aplicación del barniz de fluoruro de sodio enriquecido con fosfato tricálcico se encontró una adsorción de $2126 \pm 126$ ppm de flúor en 
esmalte desmineralizado y $455 \pm 38$ ppm en esmalte sano, así como calcio y fosfato inorgánico en concentraciones mínimas pero indispensables de detener el proceso carioso, incrementando la posibilidad de revertir la lesión incipiente..$^{21,22}$

Otros investigadores consideran que el barniz de fluoruro de sodio enriquecido con fosfato tricálcico tiene un efecto protector reducido y no encontraron ventajas significativas por su complemento cálcico. ${ }^{23,24}$ Además, dada la naturaleza multifactorial de la caries dental, la efectividad de los compuestos fluorurados se ve limitada por los determinantes biológicos y sociales.

Los escolares participantes en este estudio tenían características de alto riesgo cariogénico, lesiones de caries ya cavitadas y, no obstante la asesoría de cepillado personalizada, el porcentaje de remineralización obtenido fue bajo después de la aplicación del barniz de fluoruro de sodio enriquecido con fosfato tricálcico. Las limitaciones de esta investigación fueron: la imposibilidad de tener un control preciso en los escolares de no ingesta de alimentos en un lapso mínimo de una hora posterior a la aplicación del barniz, así como el periodo de intervención limitado a un año, para obtener resultados más contundentes sería importante extender la observación a mayor tiempo.

Los autores consideran que los programas preventivos deben incluir diferentes estrategias de promoción y protección específica del proceso carioso. Es indispensable la educación de los padres acerca de hábitos dietéticos saludables. Es recomendable la detección de las lesiones de caries incipientes, a fin de instaurar tratamientos de mínima invasión con materiales inteligentes dirigidos a la recuperación de lesiones en su fase inicial y preservación de las estructuras dentarias. La asesoría personalizada referente al cepillado dental y uso del hilo dental en combinación con la utilización de barnices fluorurados pueden cambiar el perfil epidemiológico de los escolares. La premisa fundamental para preservar las estructuras dentarias es el riguroso control de la biopelícula a través del cepillado, así como la limitación en la ingesta de alimentos y bebidas ricas en azúcares..$^{25,26}$

\section{CONCLUSIÓN}

La caries dental es un proceso donde el control de la biopelícula dentaria es determinante en la aparición y avance de la enfermedad. La acción del flúor en sinergia con compuestos cálcicos en la interfase del esmalte a través del barniz de fluoruro de sodio enriquecido con fosfato tricálcico mostró ventajas en el control de las lesiones incipientes con efecto preventivo y remineralizante, por lo que su aplicación es recomendable para coadyuvar en el control de la enfermedad.

\section{Agradecimiento}

Este estudio fue apoyado por el programa PAPIIT IN 218915.

\section{REFERENCIAS}

1. Secretaría de Salud. Resultado del Sistema de Vigilancia Epidemiológica de Patologías Bucales. SIVEPAB 203. Dic 2014 disponible en http://www.spps.salud.gob.mx/ acceso 13 septiembre del 2016.

2. Henestroza G. Caries dental. Principios y procedimientos para el diagnóstico. Lima: Universidad Peruana Cayetano, 2007:37-38.

3. Marinho VC, Worthington HV, Walsh T, Clarkson JE. Fluoride varnishes for preventing dental caries in children and adolescents (Review). Cochrane Database of Syst Rev 2013, Issue 7: CD002279. Disponible en www. cochranelibrary.com

4. Carvalho DM, Salazar M, Oliveira BH, Coutinho ES. Fluoride varnishes and decrease in caries incidence in preschool children. A systematic review. Braz J Epidemiol. 2010;13:139-49.

5. Irigoyen $\mathrm{M}$, Luengas $\mathrm{M}$, Amador Y, Zepeda M A, Villanueva T, Sánchez L. Comparación de barnices y dentífrico con flúor en la prevención de caries en escolares. Rev de Salud Pública. 2015; 17(5):801-814. 
6. Sánchez C. Desmineralización y remineralización. El proceso balance de la caries dental. Rev. ADM. 2010;67(1):30-32.

7. Secretaría de Salud. Subdirección de salud bucal. Centro Nacional de Programas Preventivos 2011.

8. Murrieta Pruneda F, Juárez López MLA. Índices epidemiológicos de morbilidad bucal en México. FES Zaragoza. UNAM 2006. ISBN 970-32-3340-6.

9. Ismail Al, Sohn W, Tellez M, Amaya A, Sen A, Hasson H, et al. The International Caries Detection and Assessment System (ICDAS): an integrated system for measuring dental Caries. Community Dent Oral Epidemiol. 2007;35:170-8.

10. Clinpro ${ }^{\mathrm{TM}}$ White Varnish keeps getting better -now with TCP. Disponible en: http://multimedia.3m.com/mws/ media/6547030/clinpro-white-varnish-extra-protectionfolder.pdf?fn=Clinpro_White_Varnish_F.pdf

11. Gil F, Aguilar M, Canamás M, Ibañez P. Periodoncia para el higienista dental. Periodoncia y osteointegración. 2005;15(1):43-58.

12. Arruda $A O$, Senthamarai Kannan $R$, Inglehart MR, Rezende CT, Sohn W. Effect of $5 \%$ fluoride varnish application on caries among school children in rural Brazil a randomized controlled trial. Communnit Dental Oral Epidemiol 2011; 40:267-76.

13. Abanto JA, Bonecker M, Raggio DP. Impacto de los problemas bucales sobre la calidad de vida de los niños. Rev Estomat Herediana.2010;20(19):38-44.

14. Marinho VC, Higgins JP, Logan S, Sheiham A. Topical fluorides (toothpastes, mouth rinses, gels or varnishes) for preventing dental caries in children and adolescents. Cochrane Database Syst Rev. 2003;4:1-164. disponible en http://www.cochranelibrary.com/

15. Featherstone JD, Domejean G. The role of remineralizing and anticaries management. Adv Dent Res. 2012;24(2):28-31.

16. Cury J, Andalo L. Enamel remineralization: controlling the caries disease or treating early caries lesions? Braz oral Res 2009;23(Spec Iss1):23-30.
17. Gao S, Zhang S, Mei M, Chin-man E, Chu Ch. Caries remineralization and arresting effect in children by professionally applied fluoride treatment -a systematic review. BMC Oral Health.2016;16-12. doi: 10.1186/s12903-016-0171-6

18. Rirattanapong $P$, Vongsavan $K$, Saengsirinavin C. Pornmahala T. Effect of fluoride varnishes containing different calcium phosphate sources on mineralization of initial primary enamel lesions. J Trop Med Public Health. 2014;45(6):1503-10.

19. Elkassas D, Arafa A. Remineralizing efficacy of different calcium-phosphate and fluoride-based delivery vehicles on artificial caries like enamel lesions. J Dent. 2014;42(4):466-74.

20. Urzúa I, Mocada G, Aranguiz V, Hoyos J, Cortes C, Ulloa MT. Efecto In vivo de la paliccaión de un barniz de flúor al 5\% sobre la colonización de los $\mathrm{S}$ mutans en el esmalte. Revista de Operatoria Dental y Biomateriales. 2006;1(2):43-46.

21. Schemehorn BR, Wood GD, McHale W, Winston AE. Comparison of fluoride uptake into tooth enamel from two fluoride varnishes containing different calcium phosphate sources. J Clin Dent. 2011;22(2):51-4.

22. Memarpour M, Soltaneimer E, Saharahmedy N. Efficacy of calcium and fluoride containing materials for the remineralization of primary teeth with early enamel lesion. Microsc Res Tech. 2015;78(9): 801-6.

23. Mohd Said SN, Ekambaram M, Yiu CK. Effect of different fluoride varnishes on remineralization of artificial enamel carious lesions. Int J Paediatric Dent 2016;27(3):163-73.

24. Shen P, Bagheri R, Walker GD, Yuan Y, Stanton DP, Reynolds $C$, Reynolds EC. Effect of calcium phosphate addition to fluoride containing dental varnishes on enamel demineralization. Aust Dent J. 2016 Sep;61(3):357-65.

25. Pita S, Fernández P, Sánchez S. Relevancia del cepillado dental y caries. Aten Primaria. 2010;42(7):372-79.

26. Molina-Frechero N, Durán-Merino D, Castañeda-Castaneira E, Juárez-López MLA. La caries y su relación con la higiene oral en preescolares. Gac Med Mex. 2015;151:485-90. 